Interleukin 21 (IL21) is a potent immunomodulatory cytokine that has been implicated in the pathogenesis of autoimmune diseases including systemic lupus erythematosus (SLE) by promoting B cell differentiation or by altering Th17 and Treg function. Dendritic cells (DCs) are potent regulators of immune responses, with mature cells promoting autoreactivity while immature immune tolerance. Whether IL21 affects DC maturation and function has not been completely addressed. Here the authors demonstrate that monocyte-derived dendritic cells (moDCs), matured in the presence of IL21, significantly increased the proliferation of allogeneic CD4 CD25 T cells compared with non-IL21-treated moDCs. The immunogenicity of IL21-treated moDCs was further increased when IL21 was present during $T$ cell activation. The enhanced $T$ cell proliferation correlated well with increased secretion of interferon (IFN) $\gamma$ and tumour necrosis factor (TNF) $\alpha$ in culture supernatants. Compared with untreated moDCs, IL21-conditioned moDCs exhibited a significant and specific downregulation of the expression of inducible co-stimulator ligand (ICOS-L). In contrast, expression of HLA-DR, CD80, CD86, CD40 and PD-L1 was not altered. Finally, maturation of moDCs in the presence of serum obtained from patients with active SLE, which has previously been reported to be rich in IL21, resulted in enhanced immunogenicity of moDCs and reduced ICOS-L expression on their surface. Experiments using neutralising antibodies of IL21 are in progress. Together these results suggest that modulation of DCs by IL21 may represent an important mechanism to explain the increased immunogenicity of DCs in autoimmune diseases.

\section{A177 INTERLEUKIN 21-CONDITIONED DENDRITIC CELLS PROMOTE T CELL REACTIVITY THROUGH THE DOWNREGULATION OF ICOS-L}

M Kavousanaki, ${ }^{1,2}$ N Orphanos, ${ }^{1,2}$ D T Boumpas, ${ }^{1,2}$ P Verginis ${ }^{1,2}{ }^{1}$ University of Crete Medical School; ${ }^{2}$ Institute of Molecular Biology and Biotechnology, Foundation for Research and Technology, Heraklion, Crete, Greece 